\title{
VIOLENCIA EN EL NOVIAZGO EN OPINIÓN DE UN GRUPO DE ADOLESCENTES DE LA SIERRA NORTE DE PUEBLA
}

VIOLENCE IN DATING IN THE OPINION OF ONE GROUP OF TEENAGES OF THE NORTHERN SIERRA OF PUEBLA

\section{RESUMEN}

La violencia es originada por múltiples factores y diversas expresiones. En el noviazgo, la violencia es un fenómeno casi imperceptible que puede confundirse con muestras de cariño y de protección. Es por ello, que este estudio tiene como objetivo analizar las opiniones de hombres y mujeres asociadas al alcance y naturaleza de la violencia en el noviazgo en adolescentes. Participaron estudiantes de tres bachilleratos ubicados en la Sierra Norte del Totonacapan en el estado de Puebla, México. La metodología empleada fue la cualitativa mediante la recolección de testimonios recabados en grupos focales, a raíz de las jornadas de vinculación con la comunidad en conjunto, con el personal de la Casa de la Mujer Indígena de Ixtepec (CAMI, A.C.). Se examinaron los tipos de violencia que describen en sus opiniones las y los adolescentes (psicológica, física y sexual) y las explicaciones que dan de cada una de ellas. Se concluye que los usos y costumbres de las y los entrevistados denotan que las prácticas de violencia se aprenden principalmente de forma no reflexiva en el hogar.

Palabras clave: Violencia, noviazgo, estudiantes, Colegio de Bachilleres, Sierra Norte de Puebla.

\section{ABSTRACT}

Violence is caused by multiple factors and diverse expressions. In dating relationship, violence is an almost imperceptible phenomenon that can be confused with the expressions of affection and protection. This study aims to analyze the opinions of men and women associated with the scope and nature of violence in teenage dating relationship. The participants were students of three high schools located in the old Northern Sierra of Totonacapan in Puebla, Mexico. The types of violence was described with the collaboration of the House of the Indigenous Woman of Ixtepex (CAMI, AC) through the application of qualitative methods of analysis which consists of the testimonies offocal groups. This was explained by women and men interviewees (psychological, physical and sexual) were examined, in some cases, after having faced some of them. We conclude that the uses and customs of the interviewees denote that the practices of violence are learned in the non-reflective way in the home, and are enhanced by the behaviors acquired through digital means.

Keywords: Violence; dating; students; highschool students; Northern Sierra of de Puebla.
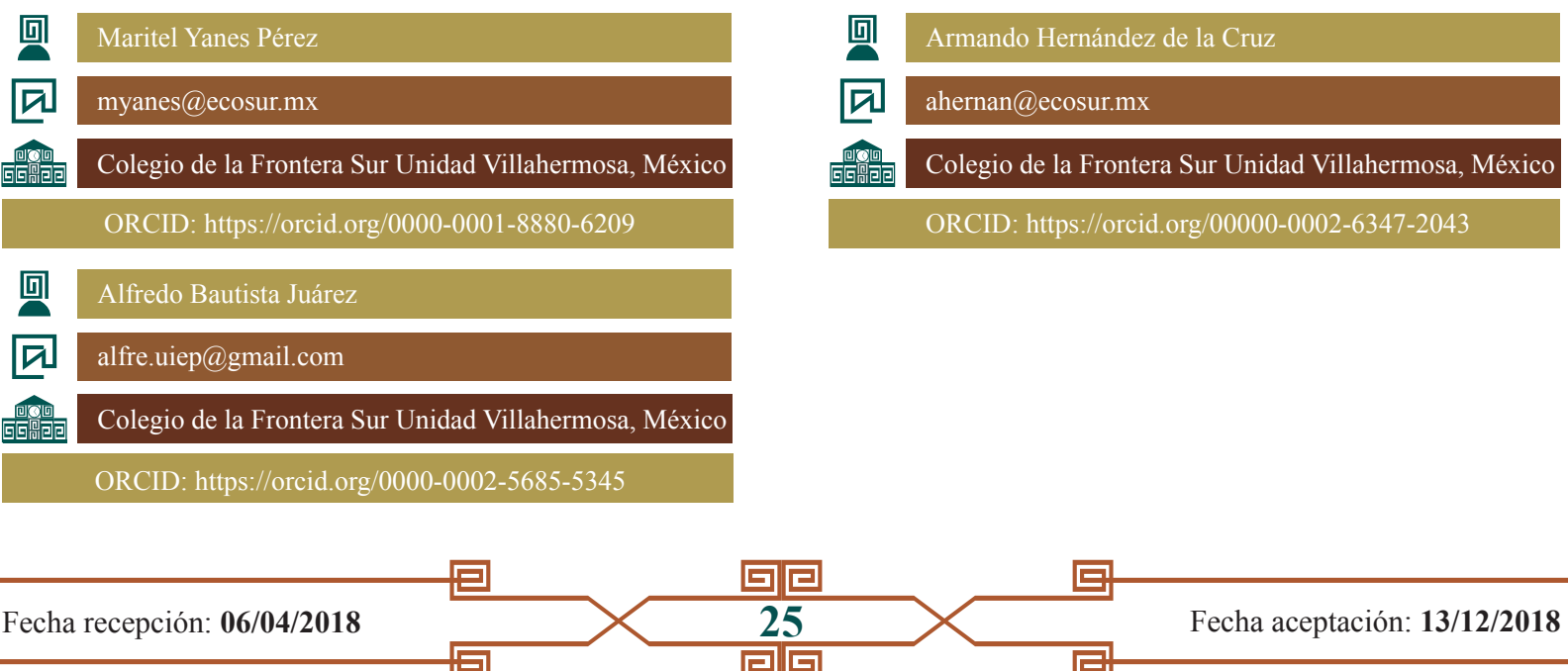


\section{INTRODUCCIÓN}

Diversos estudios han mostrado que en el período de la adolescencia se experimentan las primeras relaciones amorosas (Celis \& Rojas 2015); estas relaciones pueden devenir en conflictos y violencia en el momento en que alguno de los miembros de la pareja pretende dominar al otro, asumiendo una posición controladora o posesiva (Echeburúa \& Corral 1998; Vargas \& Barrera 2002; Linares 2006; Rubio, Carrasco \& López 2015).

Las relaciones de noviazgo entre adolescentes y jóvenes, no están libres de comportamientos encaminados a causar daño físico, psíquico o sexual a alguno de sus miembros (Organización Panamericana de la Salud 2003; Celis \& Rojas 2015; López, Lana, Rodríguez, Rodríguez \& Paino 2015). En el estudio realizado identificamos que en la opinión de los y las jóvenes entrevistadas, muchas de las conductas violentas se identifican más bien con el amor o el interés por la pareja. Esta línea de argumentación nos permite entender el concepto de violencia en parejas de adolescentes como "el conjunto de actitudes y comportamientos agresivos que se despliegan en el contexto de una relación de pareja en la que existe atracción y en la que dos miembros de la pareja se citan para salir" (Close 2005 citado por Celis \& Rojas 2015:1).

Algunos estudios han señalado que la violencia en el noviazgo puede deberse a la inexperiencia de los adolescentes. Su falta de habilidades para socializar o su poca experiencia pueden derivar en una relación agresiva. Para el caso del presente estudio detectamos que tanto hombres como mujeres entrevistados han padecido este tipo de situaciones que con el tiempo se normalizan y pueden generar patrones de conducta y de relación que se mantienen y practican hasta la edad adulta. El hecho de que tanto hombres como mujeres sufran violencia durante esta etapa no quiere decir que exista el mismo grado de gravedad en los actos violentos ejercidos, en términos de género (Celis \& Rojas 2015).

\section{Violencia en el noviazgo adolescente en México}

En la Encuesta Nacional de Violencia en las Relaciones de Noviazgo, realizada por el Instituto Mexicano de la Juventud en el año 2007, los resultados señalan que en las relaciones de noviazgo que establecen las y los jóvenes entre 15 y 24 años de edad, hay expresiones de violencia de muy distinto tipo y en diferentes grados (Instituto Mexicano de la Juventud 2008).

Los tipos de violencia identificados en dicha encuesta, fueron: la violencia física, la psicológica y la sexual. Para el primer caso se señala que el 15\% de las y los jóvenes han experimentado al menos un incidente de violencia física en la relación de noviazgo que tenían en el momento de aplicación de la encuesta (Instituto Mexicano de la Juventud 2008). En lo que respecta a la violencia psicológica, que contempla abuso emocional, humillación, maltrato verbal, entre otras, se detectó que el $76 \%$ de los jóvenes son víctimas (Instituto Mexicano de la Juventud 2008). En cuanto a la violencia sexual, las mujeres representan las dos terceras partes de las agredidas, en términos de ser forzadas o que han intentado forzarlas para mantener relaciones sexuales.

En otro estudio se analizó la prevalencia de la violencia física y psicológica en más de 7.000 estudiantes de escuelas públicas del Estado de Morelos, con edades de 11 a 24 años, quienes, habían tenido al menos una relación de pareja en su vida. En cuanto a quién ejerce la agresión, los investigadores encontraron que un 4,2\% de las mujeres y un 4,3\% de los varones, habían ejercido violencia psicológica, y que un $21 \%$ y un $19,5 \%$ de mujeres y varones, respectivamente, manifestaron haber llevado a cabo actos de violencia física. Mientras que un 7,5\% de las mujeres y un $5,5 \%$ de los varones habían ejercido tanto violencia verbal como física (Rivera, Allen, Rodríguez, Chávez \& Lazcano 2006).

Con estos dos ejemplos podemos mostrar que hay una tendencia en cuanto a, que tanto hombres como mujeres son perpetradores como víctimas de violencia, durante el noviazgo adolescente. 


\section{METODOLOGÍA}

El presente artículo de investigación se alinea dentro del paradigma de la investigación cualitativa. El estudio inició en noviembre de 2015 , en el marco de un proyecto de vinculación comunitaria; donde estudiantes de la Licenciatura en Derecho con enfoque Intercultural de la Universidad Intercultural del Estado de Puebla, se coordinaron con la Casa de la Mujer Indígena (CAMI) del municipio de Ixtepec, Puebla, para investigar sobre la violencia en el noviazgo. La recolección de la información se hizo mediante la aplicación de grupos focales cuyo interés consistió en captar la opinión de las y los adolescentes sobre el tema.

Los días 25 y 26 de abril de 2016 se visitaron tres instituciones de educación media superior de dos diferentes municipios, en las cuales se dio a conocer a las autoridades escolares y a las y los adolescentes, los objetivos de la investigación. El requisito de inclusión fue ser estudiante del colegio, sin importar si tenía una relación de noviazgo en ese momento o con anterioridad. Se les aseguró a las y los participantes su anonimato.

Se realizaron 5 grupos focales, empleando inicialmente una narración completa, partiendo de supuestos psicoproyectivos que dieron entrada a la pregunta sobre sus opiniones acerca de violencia durante el no- viazgo en su entorno escolar. Asimismo, se aplicó un cuestionario que recogía datos de identificación de los participantes. Se contó con una participación de 134 jóvenes $(60 \%$ mujeres y $40 \%$ hombres entre los 15 y 21 años), originarios de la región de la zona norte del Totonacapan del estado de Puebla, México.

En algunos grupos focales las y los estudiantes fueron participativos y tomaron con seriedad el tema tratado, la información que otorgaban la complementaban con ejemplos y la opinión de hombres y mujeres fue de forma equitativa. En general, las mujeres opinaron más que los hombres, además las participantes cuestionaron el modo de vida actual de las mujeres mostrando perspectivas de cambio. En tanto que en otros grupos focales las y los estudiantes no fueron muy comunicativos, en el momento de expresarse se notó nerviosismo y dudas cuando respondían las preguntas, aunque hombres y mujeres participaron de igual manera.

Uno de los límites del estudio es que se refiere a un contexto específico, sin embargo, los hallazgos pueden dar cuenta de contextos socioculturales referidos a los pueblos originarios.

1. Acerca de los participantes

Las edades de las y los entrevistados van de los 15 a los 21 años. La edad promedio es de 17 años. Todas las y los adolescentes, pertenecen a la región de la zona norte del Totonacapan, del estado de Puebla.

Figura 1: Distribución porcentual por sexo de las y los adolescentes de los tres bachilleratos entrevistados $(\mathrm{N}=134)$.

Distribución porcentual por sexo de las y los adolescentes de los tres bachilleratos entrevistados.

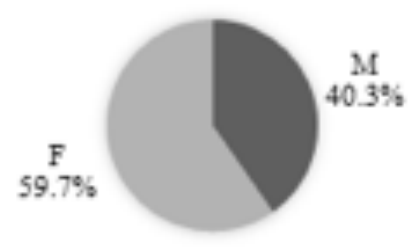

Fuente: Elaboración propia a partir de los datos arrojados por la encuesta. 
En la figura 1 se puede apreciar que casi el $60 \%$ de las personas entrevistadas son mujeres. También se logró captar la información sobre las comunidades de origen de las y los entrevistados se encontró que casi el $43 \%$ provienen de la cabecera municipal de Atlequizayan, seguido de la comunidad de Ignacio Allende con el 27\%, perteneciente al mismo municipio. Finalmente, el $10 \%$ eran originarios del municipio de Zapotitlán de Méndez. Los demás municipios y localidades están ubicados en lugares colindantes, algunos cercanos y otros lejanos a las comunidades que tienen los porcentajes más altos en la población estudiantil analizada; estas últimas son precisamente las localidades en donde se localizan los bachilleratos, en los cuales se aplicaron los grupos focales.

En las páginas siguientes se presentan los datos obtenidos en los diferentes grupos focales. Para llegar a dichos resultados se hicieron distintos procedimientos, primero se realizó la transcripción de los audios obtenidos, y después se determinó cuáles serían las categorías a profundizar. Las categorías observadas son: conceptualización de violencia, tipos, ejercicio según el género, origen, naturalización, repercusión, confianza ante la violencia experimentada y reacción ante la violencia.

Posteriormente, ya con las categorías definidas, se realizaron tablas por grupos focales clasificándolas por sexo, para separar las opiniones tanto de hombres como de mujeres. Después, con la información obtenida se identificaron las frases y opiniones que permitieron comprender la forma de pensar de las y los jóvenes de las preparatorias visitadas.

Finalmente, se redactaron las opiniones de las y los entrevistados plasmando las ideas que eran semejantes y diferentes, así como, la comparación de resultados entre ambos sexos. Esto se llevó a cabo para los cinco grupos focales. Del mismo modo, se realizó el cotejo de información obtenida de manera general de todos los grupos focales por sexo, y por último, las opiniones que fueron compartidas entre las mujeres y los hombres entrevistados. Esta información se presenta a continuación. 


\section{RESULTADOS Y DISCUSIÓN}

1. Los hallazgos: conceptualización de la violencia en el noviazgo

En la opinión de las y los adolescentes, se observan diferentes conceptualizaciones (ver tabla 1) que, esencialmente, son muy similares a las definiciones que se han reportado en estudios sobre violencia íntima en la pareja (Jackson 1999; Leen et al. 2013; Ru- bio, Carrasco \& López 2015). En los grupos, las opiniones de las y los jóvenes incluyen un rango de conductas que van desde los regaños y sermones o amenazas hasta las agresiones verbales, físicas y sexuales.

Tabla 1: Conceptualización de la violencia en las relaciones de noviazgo en estudiantes de 15 a 21 años

\begin{tabular}{|c|c|}
\hline Grupo focal & Definición \\
\hline \multirow[t]{2}{*}{$\begin{array}{l}\text { COBAEP P-24 Zapotitlán de Méndez, } \\
\text { Puebla. } \\
\text { (Primer grupo) }\end{array}$} & $\begin{array}{l}\text { Hombres: privar a su pareja de cosas, falta de } \\
\text { comunicación y confianza en la relación e } \\
\text { infidelidades. }\end{array}$ \\
\hline & $\begin{array}{l}\text { Mujeres: verbal, psicológica, física, maltratos, } \\
\text { insultos, forzamientos y que ambos sexos pueden } \\
\text { ser agresores. }\end{array}$ \\
\hline \multirow[t]{2}{*}{$\begin{array}{l}\text { COBAEP P-24 Zapotitlán de Méndez, } \\
\text { Puebla (Segundo grupo). }\end{array}$} & $\begin{array}{l}\text { Hombres: es darle miedo a la novia, agredir } \\
\text { verbalmente, tener control sobre su forma de vestir } \\
\text { y manipularla. }\end{array}$ \\
\hline & $\begin{array}{l}\text { Mujeres: la falta de respeto, confianza, sensibilidad, } \\
\text { el maltrato físico, psicológico al causarle daño a la } \\
\text { otra persona, el machismo y cuando se le restringen } \\
\text { actividades a la pareja. }\end{array}$ \\
\hline \multirow[t]{2}{*}{$\begin{array}{l}\text { Bachillerato Francisco Javier Mina, } \\
\text { Atlequizayan, Puebla. }\end{array}$} & $\begin{array}{l}\text { Hombres: cuando hay golpes, maltrato físico y } \\
\text { verbal. }\end{array}$ \\
\hline & Mujeres: llegar al extremo. \\
\hline \multirow[t]{2}{*}{$\begin{array}{l}\text { Bachillerato Tecnológico Intercultural, } \\
\text { Plantel } 002 \text { de Ignacio Allende en el } \\
\text { municipio de Atlequizayan, Puebla } \\
\text { (Primer grupo). }\end{array}$} & $\begin{array}{l}\text { Hombres: es propenso a suceder cuando un hombre } \\
\text { es machista, por la mala comunicación, es cuando } \\
\text { no se le da libertad y se le prohíbe a la otra persona } \\
\text { realizar ciertas actividades. }\end{array}$ \\
\hline & $\begin{array}{l}\text { Mujeres: es prohibirle a la pareja tener ciertas } \\
\text { amistades, en obstaculizar las cosas que a ellas les } \\
\text { gusta hacer, los celos, los golpes y los insultos. }\end{array}$ \\
\hline \multirow{2}{*}{$\begin{array}{l}\text { Bachillerato Tecnológico Intercultural, } \\
\text { Plantel } 002 \text { de Ignacio Allende en el } \\
\text { municipio de Atlequizayan, Puebla } \\
\text { (Segundo grupo). }\end{array}$} & $\begin{array}{l}\text { Hombres: es cuando el chavo maltrata a su novia y } \\
\text { es cuando empieza el bullying. }\end{array}$ \\
\hline & $\begin{array}{l}\text { Mujeres: es cuando se presentan las amenazas, } \\
\text { chantajes, maltratos y discusiones. }\end{array}$ \\
\hline
\end{tabular}

Fuente: Elaboración propia, a partir del análisis de los grupos focales. 
En la manera en que los grupos definen la violencia en las relaciones de noviazgo, es posible identificar varios elementos tales como los celos o el maltrato que están en el trasfondo de la amenaza de un daño físico, psicológico o sexual; o bien una relación de dominio de un miembro de la pareja a través de amenazas, chantajes o sermones, que constituyen tácticas para obligar y/o limitar. En cuanto al primer elemento, hay que matizar que la intencionalidad de causar daño físico, psicológico o sexual no siempre está presente en una acción violenta (Carrasco \& González 2006). Algunos autores sugieren que la auto-defensa se convierte en motivo de agresión a la pareja, al percibir que están sufriendo una agresión (Foshee et al. 2008; Straus 2008).

En el segundo elemento, el del dominio del otro miembro de la pareja, las conductas de chantaje o sermones suelen ir dirigidas a la obtención de la autoridad, así como al sometimiento de la víctima (Amor et al. 2010; Echeburúa \& Corral 1998). Finalmente, el hecho de que los golpes se produzcan duran- te una relación de noviazgo podría recuperar la opinión de aquellos adolescentes que consideran una relación y que ya han pasado por una serie de rituales, tales como pedir permiso a sus padres, lo que sugiere un vínculo más duradero e incluso con prácticas sexuales.

\section{Tipos de violencia}

En la opinión de las y los adolescentes entrevistados, quienes están involucrados en una relación de pareja experimentan diversas formas de violencia; por ejemplo en la tabla 1 podemos observar diferentes conductas violentas, así como algunas de sus combinaciones. Esto nos hace notar que las y los estudiantes en sus opiniones no sólo viven o identifican un tipo de violencia al interior de su relación o de otras relaciones de noviazgo, sino que identifican cual es el tipo de violencia predominante en las relaciones de noviazgo, entre estudiantes de los tres centros de bachillerato.

Tabla 2: Tipos de violencia en el noviazgo en estudiantes de 15 a 21 años

\begin{tabular}{|c|c|}
\hline Grupo focal & \\
\hline \multirow[t]{2}{*}{$\begin{array}{l}\text { COBAEP P-24 Zapotitlán de Méndez, } \\
\text { Puebla (Primer grupo). }\end{array}$} & $\begin{array}{l}\text { Hombres: los golpes, el bullying, daños y } \\
\text { maltratos psicológicos }\end{array}$ \\
\hline & Mujeres: el bullying y las palabras ofensivas \\
\hline \multirow[t]{2}{*}{$\begin{array}{l}\text { COBAEP P-24 Zapotitlán de Méndez, } \\
\text { Puebla (Segundo grupo). }\end{array}$} & $\begin{array}{l}\text { Hombres: el bullying, poner apodos, los daños } \\
\text { físicos, celos extremos, violaciones y muerte. }\end{array}$ \\
\hline & $\begin{array}{l}\text { Mujeres: pegarse, gritarse, celos, jaloneos, } \\
\text { manipulación, acoso y abuso. }\end{array}$ \\
\hline \multirow{2}{*}{$\begin{array}{l}\text { Bachillerato Francisco Javier } \text { Mina de } \\
\text { Atlequizayan, Puebla. }\end{array}$} & s: El maltr \\
\hline & $\begin{array}{l}\text { Mujeres: Las agresiones verbales, insultos y } \\
\text { los golpes. }\end{array}$ \\
\hline \multirow{2}{*}{$\begin{array}{l}\text { Bachillerato } \\
\text { Tecnológico Intercultural, } \\
\text { Plantel 002 de Ignacio Allende en el } \\
\text { municipio de Atlequizayan, Puebla (Primer } \\
\text { grupo). }\end{array}$} & Hombres: El bullying, las peleas y los golpes. \\
\hline & $\begin{array}{l}\text { Mujeres: las agresiones, insultos, celos, el } \\
\text { machismo y los sermones. }\end{array}$ \\
\hline \multirow{2}{*}{$\begin{array}{l}\text { Bachillerato Tecnológico Intercultural, } \\
\text { Plantel } 002 \text { de la comunidad de Ignacio } \\
\text { Allende en el municipio de Atlequizayan, } \\
\text { Puebla (Segundo grupo). }\end{array}$} & $\begin{array}{l}\text { Hombres: Es el insulto, físico, psicológico y } \\
\text { verbal. }\end{array}$ \\
\hline & $\begin{array}{l}\text { Mujeres: Los maltratos, los insultos y el } \\
\text { bullying. }\end{array}$ \\
\hline
\end{tabular}

Fuente: Elaboración propia, a partir del análisis de los grupos focales. 
Se puede observar que el enfoque del estudio de la violencia, considerando que las prácticas violentas no las ejercen solamente los hombres, es un tema relativamente inexplorado en México (Peña et al. 2013; Rivera et al. 2006; Ramírez \& Núnez 2010; Pick et al. 2010). Más aún si se trata de pueblos originarios donde el análisis de esta problemática se encuentra poco documentada.

Los cinco grupos focales entrevistados coinciden en que el ejercicio de la violencia se da tanto en hombres como en mujeres, pero es más frecuente la violencia hacia las mujeres. El análisis de nuestros resultados pone en evidencia que la violencia del hombre contra la mujer en una relación de noviazgo implica el contexto social, que en el caso de esta zona de pueblos originarios se rige por normas y valores que parten de una desigualdad de género, con roles bien definidos sobre el papel activo y dominante del hombre, o al menos es la percepción muy marcada cuando los informantes señalan que los hombres tienen el pensamiento de que se sienten superiores a ellas.

Como señalan Agoff, Rajsbaum y Herrera "esto se manifiesta no sólo al interior de la relación de pareja, sino también a través de los agentes sociales del entorno, quienes producen y reproducen cotidianamente estas concepciones de género" (2006:309). En el discurso de las mujeres se interpreta que conciben a los hombres como violentos y como más fuertes que ellas. Existe una profunda indignación por los golpes hacia las mujeres, que provocan que tengan que ser enviadas al hospital o que les causan la muerte. Ellos se atreven a pegar porque piensan que tienen más control sobre las mujeres e incluso hacia otros hombres. Casi todas las mujeres entrevistadas consideran que no son violentas. Los varones entrevistados en su mayoría reconocen que ejercen violencia, pero además revelan que entre ellos mismos las agresiones son más fuertes que hacia una mujer. En contraste, hay un grupo de hombres que declaró que la violencia existe de igual manera, es decir, que tanto varones como mujeres pueden ser agresores y que las mujeres les controlan las redes sociales.
De lo anterior se pueden destacar tres puntos. Primero, tanto para mujeres como para los varones los roles de género previamente establecidos en sus comunidades originarias continúan vigentes. La mujer sigue representando lo frágil y lo indefenso, mientras los hombres son rudos, violentos y practican dominación sobre ellas, lo que se ha nombrado como masculinidad hegemónica que es definida como "la configuración de práctica genérica que encarnan la respuesta corrientemente aceptada al problema de legitimidad del patriarcado, la que garantiza la posición dominante de los hombres y la subordinación de las mujeres" (Conell 1995:39). En ella, los varones buscan continuamente el poder, y lo ejercen sobre las mujeres, e incluso sobre otros hombres que son considerados como débiles.

El ejercicio del poder es el término clave. Para Foucault (1988) el poder se ejerce sobre las cosas y proporciona la capacidad de modificarlas, utilizarlas, consumirlas o destruirlas. Además, pone en juego relaciones entre individuos (o entre grupos), porque si se habla de estructuras o de mecanismos de poder, este designa relaciones entre parejas. Para los hombres el ejercicio del poder es una posibilidad de imponer control sobre otras personas. Una de las maneras para demostrar el poder, es mediante el uso de la sexualidad, cuándo la masculinidad se convierte en objeto de medición y comparación con otras personas que comparten la genitalidad masculina (Figueroa 2001). Otra manera de ejecutar y conservar el poder es mediante la legitimidad social del uso de la violencia física por parte de los hombres. Esta violencia se puede practicar sobre las mujeres y sobre otros hombres o grupos de hombres (Connel 1995). Ambas demostraciones de poder se presentan en la cotidianidad de las y los jóvenes entrevistados.

Segundo, el hecho que las mujeres rechacen tajantemente la violencia representa un posible cuestionamiento a esos roles de género establecidos. Esto coincide con otras investigaciones en contextos indígenas, en donde las mujeres están redefiniendo sus derechos y están empezando a debatir algunos de los 
fundamentos sobre los que se constituye el derecho indígena (como la violencia intrafamiliar), replanteando críticamente sus propios sistemas normativos (Sierra 2006; Chenaut 2007; Hernández 2010).

Por último, llama la atención que los hombres declaren que las mujeres también son violentas mientras ellas lo niegan. Esto podría deberse, por una parte, a que la agresividad es vista como un atributo masculino que no es bien aceptado y, por lo tanto, el uso de la fuerza física contra otros por parte de las mujeres está deslegitimado (Yanes 2014).

Por otra parte, se ha documentado que las mujeres ejercen el poder de manera diferente a la de los hombres. Ellas detentan el poder mediante el afecto y el cuidado erótico y maternal, también llamados poderes ocultos, con ellos logran que las necesiten. Según Bonino (1995) estos no son en realidad poderes de dominio sino pseudo poderes que imponen una reclusión en el mundo privado y representa una característica de los grupos subordinados, que consiste en manejar al superior.

Las mujeres se hacen expertas en leer las necesidades y en satisfacer los requerimientos del varón, logrando ser valorada por su eficiencia y exigiendo algunas ventajas a cambio. Sus necesidades y reclamos no pueden expresarse directamente, y por ello se hacen por vías ocultas básicamente las quejas y reproches (a los que los varones rápidamente se hacen inmunes) (Bonino 1995).

De acuerdo con lo anterior, cobra sentido que sean las mujeres las que en su mayoría vigilen el uso de las redes sociales y el celular de sus parejas, puesto que el control, la dominación y el acecho al objeto amoroso (Velázquez 2013) podrían convertirse en parte de estas estrategias silenciosas para tener injerencia directa en los cambios de conducta asociadas a mujeres y varones (Celis \& Rojas 2015). Tras las evidencias, hay que señalar que dichos comportamientos ya están presentes entre las y los adolescentes de la Sierra del Tototocapan y sin duda tendrán repercusiones sociales en sus comunidades.

\section{Origen de la violencia en el noviazgo}

Una de las teorías que habla sobre conductas agresivas aprendidas desde la psicología es la del aprendizaje social (Bandura \& Ribes 1976). En esta se plantea que las conductas pueden aprenderse por imitación y tienen como base la atención y la motivación. La primera es donde se despierta el interés de algún comportamiento ejercido por un modelo (padres, o cuidadores primarios) y el segundo es el motor que consiste en la voluntad de realizar una conducta que observamos de un modelo (Bandura \& Ribes 1976).

En nuestros resultados encontramos que las y los entrevistados mencionaron que la violencia se origina con el machismo, los problemas dentro del hogar por parte de la familia de origen, la falta de confianza y la necesidad de reconocimiento. Por lo que la dinámica familiar es un factor importante, ya que si las y los cuidadores primarios emiten conductas agresivas que llevan a cualquier tipo de violencia, está será imitada por los adolescentes llevándola a otro contexto social, en este caso en el noviazgo. También observamos que las mujeres muestran un mayor grado de tolerancia, a la violencia que son sometidas cuando perciben que su origen está en el contexto.

Puede apreciarse que la violencia es un fenómeno complejo que abarca diferentes situaciones y escenarios comunitarios, familiares y personales. La norma de lo que se considera un comportamiento aceptable o lo que constituye un daño para otras personas ha variado en diferentes épocas y culturas (Pick, Leenen, Givaudan \& Prado 2010). En el siguiente cuadro se presentan las opiniones de las y los adolescentes sobre el origen de la violencia en el noviazgo. 
Tabla 3: Origen de la violencia en el noviazgo en estudiantes de 15 a 21 años.

\begin{tabular}{|c|c|}
\hline Grupo focal & Origen \\
\hline \multirow[t]{2}{*}{$\begin{array}{l}\text { COBAEP P-24 de Zapotitlán de } \\
\text { Méndez, Puebla (Primer grupo). }\end{array}$} & $\begin{array}{l}\text { Hombres: por privar a su pareja de cosas, falta de } \\
\text { comunicación y confianza en la relación. }\end{array}$ \\
\hline & Mujeres: por los celos y porque no hay confianza. \\
\hline \multirow[t]{2}{*}{$\begin{array}{l}\text { COBAEP P-24 de Zapotitlán } \\
\text { Méndez, Puebla (Segundo grupo). }\end{array}$} & $\begin{array}{l}\text { Hombres: en el ejemplo que da la familia, en la tele } \\
\text { e inclusive con los amigos. }\end{array}$ \\
\hline & \\
\hline \multirow[t]{2}{*}{$\begin{array}{l}\text { Bachillerato Francisco Javier Mina de } \\
\text { Atlequizayan, Puebla. }\end{array}$} & $\begin{array}{l}\text { Hombres: es por la desconfianza o porque aún no } \\
\text { conocen de esta cuestión. }\end{array}$ \\
\hline & $\begin{array}{l}\text { por la falta de comunicación, de cariño y } \\
\text { elos. }\end{array}$ \\
\hline \multirow{2}{*}{$\begin{array}{l}\text { Bachillerato Tecnológico Intercultural, } \\
\text { Plantel } 002 \text { de Ignacio Allende en el } \\
\text { municipio de Atlequizayan, Puebla } \\
\text { (Primer grupo). }\end{array}$} & $\begin{array}{l}\text { lel hogar por parte de la } \\
\text { mbrados" }\end{array}$ \\
\hline & $\begin{array}{l}\text { Mujeres: proviene dentro del hogar por parte de la } \\
\text { familia, por la educación que dan los padres. }\end{array}$ \\
\hline \multirow{2}{*}{$\begin{array}{l}\text { Bachillerato Tecnológico Intercultural, } \\
\text { Plantel } 002 \text { de la comunidad de } \\
\text { Ignacio Allende en el municipio de } \\
\text { Atlequizayan, Puebla (Segundo } \\
\text { grupo). }\end{array}$} & Hombres: cuando empieza el bullying. \\
\hline & $\begin{array}{l}\text { Mujeres: se genera por los celos, desconfianza, } \\
\text { machismo y traición. }\end{array}$ \\
\hline
\end{tabular}

Fuente: Elaboración propia, a partir del análisis de los grupos focales.

Sobre esta categoría los varones opinaron que la violencia en el noviazgo se genera cuando empiezan a privar a su pareja de cosas, con el bullying, la falta de comunicación y la confianza en la relación. Las mujeres piensan que todos esos factores influyen, pero agregaron que los celos, el machismo y la traición también originan violencia en el noviazgo. Tanto hombres como mujeres coinciden que estos aprendizajes se adquieren dentro del hogar por parte de la familia. Sólo los varones mencionaron que también influyen los amigos o incluso la televisión.

Estos hallazgos coinciden con los resultados de otras investigaciones realizadas en México, donde se ha resaltado el estilo de crianza como un factor importante, pues establece un comportamiento diferenciado para los hombres y las mujeres: autoritario en los primeros y abnegado en las segundas (Ramírez \& Núnez 2010), ambos sexos "son configurados para adoptar y representar los tipos de violencia en el momento en que los jóvenes inician una relación de pareja, en- tonces dichos patrones son normalizados y validados como formas de común convivencia en la sociedad" (Olvera, Arias \& Amador 2012:167).

\section{En otros países}

Los factores de riesgo de la violencia en las parejas jóvenes que han sido más estudiados son la observación de violencia entre los padres, la aceptación de la violencia en la relación de pareja, tener amigos o conocidos que han sido víctimas o victimarios de dicha violencia, los roles tradicionales de género y la experiencia de haber sido víctima de violencia por parte de la pareja o en la familia de origen (Rey 2008:232). 


\section{Naturalización de la violencia}

En la tabla 4, se observa que la violencia masculina es percibida como un castigo hacia la mujer por el no cumplimiento del rol de género femenino. En investigaciones previas se ha documentado que la violencia es considerada como algo normal en las relaciones amorosas de noviazgo por muchos adolescentes (Martínez 2014), veamos cómo la interpretan las y los estudiantes entrevistados.

Tabla 4: Naturalización de la violencia en el noviazgo en estudiantes de 15 a 21 años.

\begin{tabular}{|c|c|}
\hline Grupo focal & Asimilación \\
\hline \multirow[t]{2}{*}{$\begin{array}{l}\text { COBAEP } \quad \text { P-24 } \quad \text { Zapotitlán } \\
\text { Méndez, Puebla (Primer grupo). }\end{array}$} & $\begin{array}{l}\text { Hombres: "es parte dé, porque va en el sistema del } \\
\text { noviazgo"; "La violencia no es normal" }\end{array}$ \\
\hline & Mujeres: la violencia no es normal \\
\hline \multirow{2}{*}{$\begin{array}{l}\text { COBAEP P-24 de Zapotitlán de } \\
\text { Méndez, Puebla (Segundo grupo). }\end{array}$} & Hombres: las personas involucradas están mal \\
\hline & $\begin{array}{l}\text { Mujeres: si ellas lo permitieran sería violencia en sí } \\
\text { misma, porque es su cuerpo y deben de respetarse y } \\
\text { más si es sin su consentimiento. }\end{array}$ \\
\hline \multirow[t]{2}{*}{$\begin{array}{l}\text { Bachillerato Francisco Javier Mina } \\
\text { de Atlequizayan, Puebla. }\end{array}$} & $\begin{array}{l}\text { Hombres: La violencia no es normal. "El hombre } \\
\text { llega hasta donde la mujer lo permite". "La mujer } \\
\text { debe de hacer lo que el hombre quiere". }\end{array}$ \\
\hline & $\begin{array}{l}\text { Mujeres: "se supone que él no tiene derecho a } \\
\text { maltratarte, te tiene que tratar bien". }\end{array}$ \\
\hline \multirow{2}{*}{$\begin{array}{l}\text { Bachillerato } \\
\text { Intercultural, Plantel } 002 \text { de Ignacio } \\
\text { Allende en el municipio de } \\
\text { Atlequizayan, Puebla (Primer grupo). }\end{array}$} & $\begin{array}{l}\text { Hombres: si uno está acostumbrado a llevarse así con } \\
\text { la otra persona no se deben de molestar, pero, si no se } \\
\text { llevan de esa manera, pues no lo deben de hacer. }\end{array}$ \\
\hline & $\begin{array}{l}\text { Mujeres: hay que darse a respetar, la mujer pierde } \\
\text { más porque es discriminada y señalada por la misma } \\
\text { sociedad. "Una mujer, tal vez, podemos llegar a } \\
\text { confundir esa violencia en exceso de amor"; "no } \\
\text { confundir las prohibiciones, de que hay me está } \\
\text { cuidando porque me quiere, o hay es que sólo me } \\
\text { quiere sólo para él o cosas así" }\end{array}$ \\
\hline \multirow[b]{2}{*}{$\begin{array}{l}\text { Bachillerato } \\
\text { Tecnológico } \\
\text { Intercultural, Plantel } 002 \text { de la } \\
\text { comunidad de Ignacio Allende de } \\
\text { Atlequizayan, Puebla (Segundo } \\
\text { grupo). }\end{array}$} & Hombres: la violencia no está bien \\
\hline & $\begin{array}{l}\text { Mujeres: dime que no te gusta y yo te digo que no me } \\
\text { gusta }\end{array}$ \\
\hline
\end{tabular}

Fuente: Elaboración propia, a partir del análisis de los grupos focales.

En los varones encontramos diferentes opiniones. Algunos piensan que la violencia no es normal, mientras la gran mayoría manifiesta que es parte del sistema del noviazgo, que es una costumbre, que las mujeres lo permiten y que ellas deben de hacer lo que el hombre les dice.

En cambio, casi todos los grupos de mujeres ven a la violencia como algo fuera de lo normal. Sin embargo, en un grupo se observó el castigo social que se le impone a la mu- jer por no darse a respetar, opinaron que la mujer pierde más, porque es discriminada y señalada por la misma sociedad. Agregaron que a veces las mujeres confunden las prohibiciones o los celos con amor.

Se observa que nuevamente los estereotipos o roles de género tradicionales están presentes en las y los estudiantes entrevistados. Y en el caso de las mujeres están en una constante redefinición de su papel ante la sociedad. 


\section{Repercusión de la violencia en el no- viazgo}

En este apartado se describen las consecuencias del maltrato narradas por las y los jóvenes, así como las relaciones existentes entre unas y otras, limitándonos a las que han resultado ser más significativas.

La mayoría de las mujeres identifican aspectos relacionados con la depresión, el pánico, la desconfianza, la inseguridad y el temor a las relaciones futuras. Además, piensan que es una situación que siempre será así e interiorizan esa idea. Estos aspectos generan a las víctimas un grado importante de inadaptación a la vida cotidiana y se refleja en lo que podemos denominar un malestar emocional, que repercute en síntomas depresivos.

En tanto que algunos varones opinan que la violencia en el noviazgo genera miedo, humillación, baja autoestima, temores, traumas, conflictos e inseguridad en la toma de decisiones, es posible deducir que una considerable cantidad de las y los jóvenes entrevistados muestran esas características y, atribuyen a ellas los bajos niveles de estrategias para afrontar los actos de violencia en el noviazgo.

Los factores que inicialmente se presentan como un juego pero que potencialmente derivan en situaciones de violencia, con repercusiones de daño que los y las jóvenes perciben como: uso de la fuerza, miedo, traumas, baja autoestima, depresión y desconfianza.

\section{Confianza (búsqueda de ayuda) ante la presencia de violencia en el noviazgo}

Con sus particularidades y semejanzas, los pueblos originarios a los que pertenecen las y los entrevistados, representan una muestra del escenario cultural en el que se desenvuelven las mujeres afectadas por la violencia en su noviazgo.

En los tres planteles donde se realizó el estudio, la violencia en el noviazgo no ha sido reconocida por las autoridades como un problema de salud pública. Por otra parte las afectadas no ven a la institución educativa como un canal para resolver asuntos de pareja, que se consideran de orden personal y privado; lo que invisibiliza muchas situaciones y prácticas violentas, que al irse estableciendo se van normalizando.

Por otro lado, en los Colegios de Bachilleres estudiados, no se han incorporado acciones de prevención o de atención a la violencia en el noviazgo en términos institucionales. Las y los jóvenes afectados reciben algo de apoyo en función del interés individual de los y las profesoras u orientadores, quienes no tienen una capacitación específica para apoyar en estos casos.

Ante este contexto, los varones participantes en los grupos focales comentan que cuando buscan ayuda, recurren a sus familias en caso de padecer algún tipo de violencia, en específico a sus padres o hermanos; pero también a su mejor amigo o a algún maestro. Mientras que las mujeres, la mayoría de las entrevistadas si sufrieran de violencia: se lo contarían a alguien de confianza, amiga o amigo, a su familia, en especial a su madre, o a alguien mayor. Aunque también existió la opinión de que lo reflexionaría por sí misma. En este sentido, los resultados encontrados en las opiniones que se analizaron, muestran que el contexto patriarcal y la inequidad de género juegan un papel importante en invisibilizar la violencia en las relaciones de pareja, durante el noviazgo; la falta de reconocimiento del problema en muchas ocasiones es un factor decisivo para no buscar ayuda; ya que se vive en una situación que se considera normal.

\section{Reacción ante la violencia en el noviaz-} go

Las y los jóvenes mencionan como importante establecer una distancia con los agresores; darse un espacio y reflexionar sobre la situación que viven. Esta toma de acciones permite que ubiquen que esta situación puede atenderse en un ámbito más allá de la pareja, y que, en dado caso, es susceptible de llevarse ante las autoridades competentes. El tener valor en pedir apoyo a las autoridades, denunciar y contárselo a personas de 
confianza, darse un pensamiento de lo que quieres y lo que no quieres, son las reacciones que proponen los y las jóvenes de la Sierra Norte de Puebla que entrevistamos.

Los varones manifestaron las siguientes reacciones ante la violencia en el noviazgo: alejarse, aislarse, solicitar consejos, informarse, respetarse, denunciar, pedir apoyo a las autoridades, contarle a alguna de la persona de su confianza, o decirles a sus padres. En general, los testimonios de ambos géneros nos muestran transformaciones en la manera de ver la violencia en el noviazgo; indudablemente este posicionamiento es un referente para las comunidades de origen de los y las participantes, lo que supone que es posible una respuesta social más adecuada a estos casos de violencia. Aunque es importante señalar que los contextos no necesariamente asimilan estos posicionamientos, en la medida de que los usos y costumbres se arraigan como comportamientos normales, lo que en muchas ocasiones justifica la violencia entre las relaciones de pareja, la inequidad entre géneros y se reproduce como una práctica socialmente tolerada o abiertamente aceptada.

\section{CONCLUSIONES}

La violencia en el sistema del noviazgo, es un tema de alta complejidad, por lo que su abordaje conlleva a analizar prácticas sociales y étnicas. Este estudio plantea un acercamiento a la comprensión de esta problemática.

Para las y los adolescentes entrevistados la violencia en el noviazgo es un rango de conductas que van desde los sermones, las amenazas y los celos hasta las agresiones verbales, físicas y sexuales. El ejercicio de la violencia que se lleva a cabo dentro de una relación es cosa de ambos, tanto de hombres como de mujeres, pero es más frecuente la violencia hacia las mujeres.

El manejo de violencia dentro del noviazgo está sufriendo cambios culturales debido a la información que les viene de fuera (televisión y redes sociales). Sin embargo, estos cambios en realidad no producen grandes alteraciones a las prácticas culturales, es decir, no modifican del todo los usos y costumbres. Tanto para mujeres como para los varones adolescentes de la Sierra Norte del Totonacapan los roles de género previamente establecidos en sus comunidades originarias continúan vigentes; pero al mismo tiempo, algunas mujeres están empezando a cuestionarlos.

Tanto hombres como mujeres tienen una interpretación particular acerca de qué es la violencia y cómo se ejerce. Casi todos los varones manifiestan que la violencia es una costumbre y que forma parte del sistema del noviazgo. Mientras las mujeres, piensan que la violencia no se debe de permitir, pero ellas mismas se auto-castigan por no darse a respetar y toman pocas acciones para cambiar su condición. Entonces, ellas están en una constante redefinición de su papel ante la sociedad.

El análisis de la información permitió diferenciar como parte de las razones de la violencia percibidas por las mujeres entre: motivos y causas. El estilo de crianza es un factor que influye en la violencia en el noviazgo, ambos sexos son configurados para 
adoptar y representar los tipos de violencia en el momento en que las y los jóvenes inician una relación de pareja.

Las consecuencias del maltrato narradas por las y los jóvenes revelan que quien es tratado con violencia percibe una carencia de posibilidades para modificar su vida y controlar su futuro. Los factores que sobresalen en este estudio son: la baja autoestima y el estilo de crianza. La violencia en el noviazgo no ha sido reconocida por las autoridades de estas comunidades indígenas como un problema de salud pública. Tampoco los Colegios de Bachilleres estudiados se han preocupado por incorporar acciones de prevención o de atención a la violencia en el noviazgo dentro de su quehacer cotidiano. El peso principal de tratar de hablar lo que ocurre con la relación violenta en el noviazgo recayó sobre las jóvenes mujeres mientras que la mayoría de los varones prefieren guardar silencio.

Como medida preventiva consideramos que desde la niñez debe fomentarse la igualdad $\mathrm{y}$ el respeto entre hombres y mujeres, y que en esta tarea no solo se involucre a la comunidad estudiantil y al profesorado sino también a las madres y padres de familia. Para la atención de la violencia en el noviazgo entre adolescentes, los profesionales de la salud y la educación deben establecer medidas de intervención para la solución del problema para disminuir la violencia durante el noviazgo y evitar su progresión hacía la vida adulta.

Por último, nos parece importante agregar, que se necesitan más estudios que aborden la violencia en el noviazgo desde la perspectiva de género, y que analicen además como las tecnologías de información y la crisis de familia han impactado a las sociedades en general y a las comunidades indígenas en específico, por lo que se abre el debate y se incentiva a que se realicen más investigaciones referentes al tema.

\section{REFERENCIAS BIBLIOGRÁFICAS}

Agoff, C., Rajsbaum, A. \& Herrera, C. (2006). Perspectivas de las mujeres maltratadas sobre la violencia de pareja en México. Salud pública de México, 48 (2), 307-314.

Amor, P., Corral, P., Bohórquez, I., Oria, J., Rodríguez, M., López, F. \& Calderón, D. (2010). Violencia de género y adicción a drogas en Centros de Día. Sevilla, España: Junta de Andalucía.

Bandura, A. \& Ribes, E. (1975). Modificación de la conducta. Análisis de la agresión y la delincuencia. México, D.F.: Trillas.

Bonino, L. (1995). Micromachismos: La violencia invisible en la pareja (versión corregida y ampliada). Jornadas de la Federación de sociedades españolas de terapia familiar. Madrid, España. Recuperado de https://es.scribd. com / doc/39560822 / Bonino-Mendez-Luis-Micromachismos-La-violencia-invisible-en-la-pareja

Carrasco, M. \& González, M. (2006). Aspectos conceptuales de la agresión: definición y modelos explicativos. Acción Psicológica, 4(2), 7-38.

Celis, A. \& Rojas, J. L. (2015). Violencia en el noviazgo desde la perspectiva de varones adolescentes. Informes psicológicos, 15(1), 83-104.

Chenaut, V. (2007). Género y antropología jurídica en México. Papeles de Trabajo, (15), 47-72.

Conell, R. (1995). La organización social de la masculinidad. En T. Valdés \& J. Olavarría(ed.). Masculinidad/es poder y crisis (pp. 31-48). Santiago de Chile: FLACSO-ISIS Internacional, ediciones de las mujeres \# 24.

Echeburúa, E. \& Corral, P. (1998). Manual de violencia familiar. Madrid: Siglo XXI.

Figueroa, J. (2001). Los procesos educativos como recurso para cuestionar modelos hegemónicos masculinos. Diálogo y debate de cultura politica (Número especial sobre Política y Género) (15-16), 7-32. 
Foshee, A., Karriker-Jaffe, J., Reyes, L., Ennett, T., Suchindran, C., Bauman, E. \& Benefield, S. (2008). What accounts for demographic differences in trajectories of adolescent dating violence? An examination of intrapersonal and contextual mediators. Journal of Adolescent Health, 42(6), 596-604.

Foucault, M. (1988). El sujeto y el poder. Revista Mexicana de Sociología, 50(3,), pp. 3-20.

Hernández, A. (2010). Multiculturalismo y género. En Ohmstede A., Salmerón F., Valladares L. \& Escamilla M.G. (coords.). Reformas del Estado. Movimientos Sociales y Mundo Rural en América Latina en el siglo XX. México: UNAM-COLMEX, CIESAS, COLMICH, UAM, UIA, CEAS.

Instituto Mexicano de la Juventud (2008). Encuesta Nacional de Violencia en las relaciones de Noviazgo 2007. Resumen Ejecutivo. México. Recuperado de http://www.imjuventud.gob. $\mathrm{mx} /$ imgs/uploads/ENVINOV_2007_-_Resultados_Generales_2008.pdf

Jackson, M. (1999). Issues in the Dating Violence Research: A Review of the Literature. $A g$ gression and Violent Behavior, 4(2), 233-247.

Leen, E., Sorbring, E., Mawer, M., Holdsworth, E., Helsing, B. \& Bowen, E. (2013). Prevalencem, dinamic risk factors and the efficacy of primary interventions for adolescent dating violence: An international review. Aggression and Violent Behavior, 18(1), 159. 174.

Linares, L. (2006). Las formas del abuso. La violencia fisica y psíquica en la familia y fuera de ella. Barcelona, España: Paidós Ibérica.

López, J., Lana, A., Rodríguez L., Rodríguez J. \& Paino, G. (2015). Percepción y etiquetado de la experiencia violenta en las relaciones de noviazgo juvenil. Gaceta Sanitaria, 29(1), $21-26$

Martínez A. (2014). Prevención de violencia en el noviazgo: una revisión de programas publicados entre 1990 y 2012. Pensamiento Psicológico, 12(1), 117-132.

Olvera, A., Arias, J. \& Amador, R. (2012). Tipos de violencia en el noviazgo: Estudiantes universitarias de la UAEM, Zumpango. Revista Electrónica de Psicología Iztacala, 15(1), 150-170.
Organización Panamericana de la Salud (2003). La violencia, un problema mundial de salud pública. En Krug, E., Dahlberg, L., Mercy, J., Zwi, A. \& Lozano R. (eds.). Informe mundial sobre la violencia y la salud (pp. 3-24). Washington D.C.: Organización Mundial de la Salud. Recuperado de https:// es.scribd.com/document/246049288/violencia-juvenil

Peña, F., Zamorano, B., Hernández, G., Hernández, M., Vargas, J. \& Parra, V. (2013). Violencia en el noviazgo en una muestra de jóvenes mexicanos. Revista Costarricense de Psicología, 32(1), 27-40.

Pick, S., Leenen, I., Givaudan, M. \& Prado, A. (2010). "Yo quiero, yo puedo... prevenir la violencia": Programa breve de sensibilización sobre violencia en el noviazgo. Salud Mental, 33(2), 145-152.

Ramírez, A. \& Núnez, A. (2010). Violencia en la relación de noviazgo en jóvenes universitarios: Un estudio exploratorio. Enseñanza $e$ Investigación en Psicología, 15(2), 273-283.

Rey A. (2008). Prevalencia, factores de riesgo y problemáticas asociadas con la violencia en el noviazgo: Una revisión de la literatura. Avances en Psicología Latinoamericana, 26(2), 227-241.

Rivera, L., Allen, B., Rodríguez, G., Chávez, R. \& Lazcano, E. (2006). Violencia durante el noviazgo, depresión y conductas de riesgo en estudiantes femeninas (12-24 años). Salud Pública de México, 48 (supl. 2), 288-296.

Rubio, F., Carrasco, M. \& López, M. (2015). Factores asociados a la violencia entre adolescentes: Una revisión crítica. Anuario de Psicología Jurídica, 25(1), 47-56.

Sierra, M. (2006). El multiculturalismo en disputa: Derechos Humano, Género y Diversidad Cultural. Grandes Temas de la Antropología Jurídica. (pp. 59-78). Oaxtepec, Morelos: Red Latinoamericana de Antropología Jurídica. Recuperado de http://sgpwe.izt.uam.mx/ files/users/uami/lauv/Grandes_Temas_ Antropologia_Juridica.pdf

Straus, M. (2008). Dominance and simmetry in partner violence by male and female university students in 32 nations. Children and 
Youth Services Review, 30(3), 252-275.

Vargas, E. \& Barrera, F. (2002). Adolescencia, relaciones románticas y actividad sexual: Una revisión. Revista Colombiana de Psicología, (11), 115-134.

Velázquez, M. (2013). Violencia on line en estudiantes de licenciatura (México). V Congreso Iberoamericano de Violencia Escolar. Santiago de Chile.

Yanes, M. (2014). Estudio del homicidio en México durante la primera década del siglo XXI desde la perspectiva de género. Una profundización desde el caso de Tabasco. (Tesis de doctorado), El Colegio de México, México.
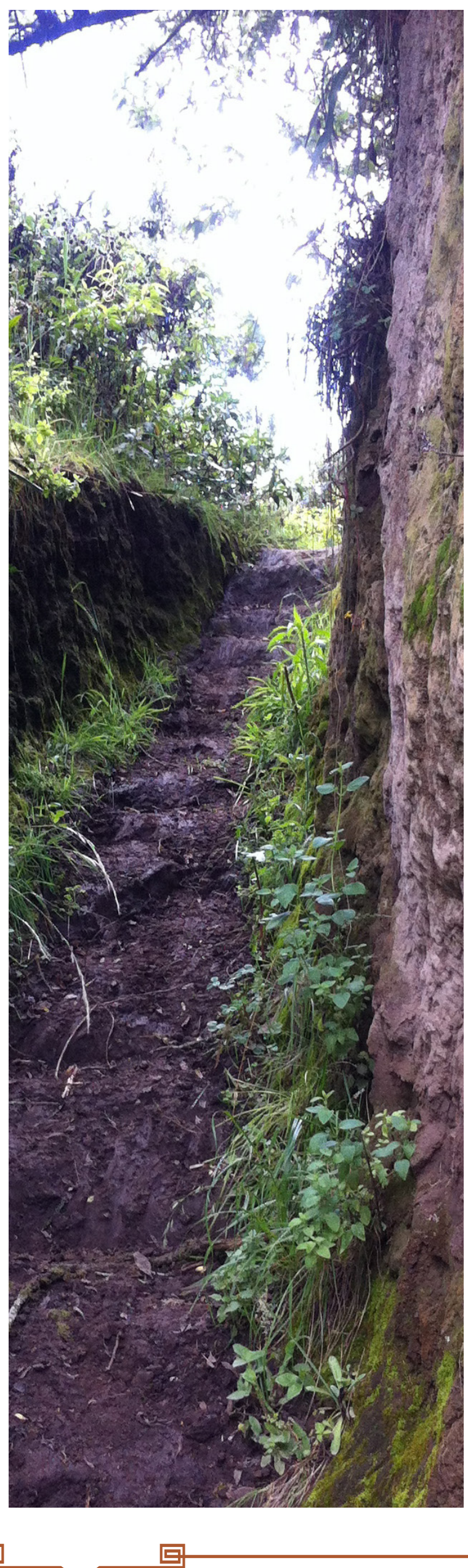